University of Nebraska - Lincoln

DigitalCommons@University of Nebraska - Lincoln

\title{
Background-Free, Quasi-Monoenergetic Electron Beams from a Self-Injected Laser Wakefield Accelerator
}

\author{
Sudeep Banerjee \\ University of Nebraska-Lincoln, Sudeep.Banerjee@asu.edu \\ Serguei Y. Kalmykov \\ University of Nebraska-Lincoln, s.kalmykov.2013@ieee.org \\ Nathan D. Powers \\ University of Nebraska-Lincoln, ndp5@byu.edu \\ Viswanathan Ramanathan \\ University of Nebraska - Lincoln, ramanath@cse.unl.edu \\ Nathan A. Chandler-Smith \\ University of Nebraska - Lincoln, nchandlersmith2@unl.edu
}

See next page for additional authors

Follow this and additional works at: https://digitalcommons.unl.edu/physicsumstadter

Part of the Physics Commons

Banerjee, Sudeep; Kalmykov, Serguei Y.; Powers, Nathan D.; Ramanathan, Viswanathan; Chandler-Smith, Nathan A.; Brown, Kevin J.; Chen, Shuo; Moorthi, A.; Ghebregziabher, I.; Maharjan, C.; Shadwick, Bradley Allan; Umstadter, Donald; Beck, A.; Lefebvre, E.; Cowan, A.; and Bruhwiler, D., "Background-Free, QuasiMonoenergetic Electron Beams from a Self-Injected Laser Wakefield Accelerator" (2011). Donald Umstadter Publications. 80.

https://digitalcommons.unl.edu/physicsumstadter/80

This Article is brought to you for free and open access by the Research Papers in Physics and Astronomy at DigitalCommons@University of Nebraska - Lincoln. It has been accepted for inclusion in Donald Umstadter Publications by an authorized administrator of DigitalCommons@University of Nebraska - Lincoln. 


\section{Authors}

Sudeep Banerjee, Serguei Y. Kalmykov, Nathan D. Powers, Viswanathan Ramanathan, Nathan A. ChandlerSmith, Kevin J. Brown, Shuo Chen, A. Moorthi, I. Ghebregziabher, C. Maharjan, Bradley Allan Shadwick, Donald Umstadter, A. Beck, E. Lefebvre, A. Cowan, and D. Bruhwiler 


\title{
Background-Free, Quasi-Monoenergetic Electron Beams from a Self-Injected Laser Wakefield Accelerator
}

\author{
S. Banerjee, S. Kalmykov, N. Powers, V. Ramanathan, N. C.-Smith, K. Brown, S. Chen, A. Moorthi, \\ I. Ghebregziabher, C. Maharjan, B. Shadwick, D. Umstadter \\ Department of Physics and Astronomy, University of Nebraska, Lincoln, NE 68516, USA \\ A. Beck, E. Lefebvre \\ CEA, DAM, DIF, 91297 Arpajon Cedex, France \\ A. Cowan, D. Bruhwiler \\ Tech-X Corporation, 5621 Arapahoe Avenue, Suite A, Boulder, CO 80303, USA \\ Presenting author e-mail address: sudeep@hfsserve.unl.edu
}

\begin{abstract}
Stable 200-400-MeV quasi-monoenergetic electron bunches $(\Delta \mathrm{E} / \mathrm{E}<10 \%), \sim 10-\mathrm{pC}$ charge, and no dark-current are produced when a self-injected laser plasma accelerator is optimized. PIC simulations demonstrate these beams are produced near the threshold for selfinjection.
\end{abstract}

\section{Introduction}

Progress in the technology of short laser pulse amplification has made it possible for laser-plasma accelerators (LPAs) to generate quasi-monoenergetic electron beams in the laboratory [1-3] and to approach the GeV energy range [4-8]. Experiments show a direct correlation between the generation of collimated electron beams and formation of a unique plasma structure -- an electron density "bubble" -- trailing a relativistically intense laser pulse $[9,10]$. The bubble forms behind the driver when the laser ponderomotive force creates complete electron cavitation (due to their high inertia, fully stripped ions remain immobile). Nonlinear evolution of the driver causes variations in the bubble shape, which triggers injection of ambient plasma electrons and forming a collimated electron beam [11]. In order to meet the stringent requirements of applications, such as laser-plasma based x-ray sources [12] LPAs must produce high-energy, quasi-monoenergetic electron beams without a largedivergence, low energy, polychromatic background (dark-current). This is important to minimize the noise produced in interactions, and improve the quality of secondary x-ray radiation. Also, poorly collimated background electrons represent a significant radiation hazard (large dose) when an LPA operates at high repetition rates. The additional shielding required offsets one of the important advantages associated with LPAs.

To reduce electron energy spread and eliminate the low-energy tails, continuous self-injection (the source of dark current) must be suppressed via proper optimization of the driver evolution, and controlling the laser pulse and target conditions. Despite significant recent progress, most reports still show electron beams with the quasimonoenergetic feature on top of a broad distribution spanning the range from a few $\mathrm{MeV}$ to the maximum energy observed [13]. We have demonstrated stable, dark-current-free, 200--400 MeV electron beams with narrow energy spread, generated in the bubble regime, near the self-injection threshold. In our work, selfinjection is controlled by restricting the location where self-focusing and bubble formation occurs, in a finite length plasma. In prior studies, injection was controlled by keeping the laser power below the self-focusing threshold. It is also shown that a moderate plasma density of $5-8 \times 10^{18} \mathrm{~cm}^{-3}$, and a high-quality laser pulse, reduces the risk of filamentation, makes the accelerator controllable, and enables generation of high-quality electron beams over a large energy range. Features such as tunability of the beam energy, and stable operation, are critical for applications [14].

\section{Results and discussion}

The experiments were carried out with the 100-TW laser DIOCLES. The system produces linearly polarized pulses with a central wavelength $\lambda=805 \mathrm{~nm}$, maximum energy $3.5-\mathrm{J}$ at $10-\mathrm{Hz}$ repetition rate, and pulse duration $\tau_{\mathrm{L}}=30 \mathrm{fs}(\mathrm{FWHM})$. The laser beam is incident on a deformable mirror that operates in a feedback loop with a wavefront sensor. The $70-\mathrm{mm}$ diameter laser pulse with spatial aberrations corrected, is focused to a nearly diffraction limited spot (Strehl ratio $~ 0.95$ ) using a one meter focal length dielectric-coated off-axis paraboloid. The intensity profile in the central spot allows a Gaussian fit with a radius $r_{0}=13.6 \mu \mathrm{m} ; 70 \%$ of incident energy is contained within a diameter $2 \mathrm{r}_{0}$. The work reported here was performed with $30--60 \mathrm{TW}$ on target. The laser pulse was spatially and temporally characterized at full power using a multi-stage beam sampling system. The shot-to-shot pointing stability of the laser pulse on target was $\pm 7 \mu \mathrm{rad}$.

The target is a high-density jet of neutral helium produced by a 3 or $4 \mathrm{~mm}$ diameter supersonic Laval nozzle. The neutral density profile (characterized interferometrically) has symmetric downramps along the edges extending over $0.5 \mathrm{~mm}$, and a 2 or $3 \mathrm{~mm}$ central flat region. The laser pulse is focused before the front edge of 
the nozzle. The focused pulse fully ionizes the medium producing a plasma. Images of the plasma emission at $800 \mathrm{~nm}$ show a uniform laser-created plasma column spanning the entire jet length. The accelerated electrons exiting the plasma impinge on a fluorescent screen (LANEX) that is imaged with a 12-bit CCD. The absolute response of LANEX to electrons, calibrated using an $18 \mathrm{MeV}$ radio-frequency linear accelerator (Siemens Primus), is used to obtain the charge in a specified energy interval. Electron energy is measured using a magnetic spectrometer operating in a slit-free geometry and having a range of 20 -- $500 \mathrm{MeV}$.

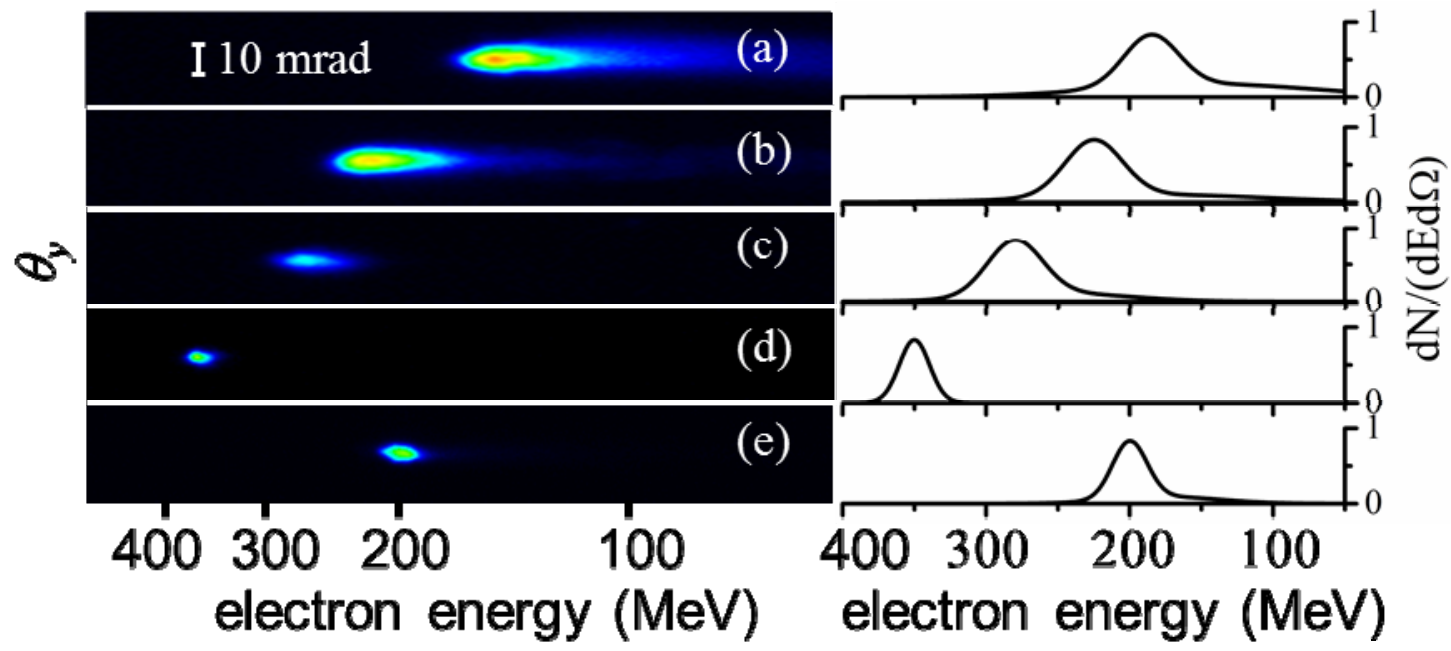

Figure 1: Spectrally dispersed electron beams at the exit of magnetic spectrometer and corresponding lineouts of electron spectra deconvolved using the GPT code taking into account the spectrometer response function and electron beam divergence. Statistics and brightness for these beams are shown in Table 1 . Panels (a) -- (e) correspond to plasma densities $12 ; 9 ; 7.2 ; 6 ;$ and $4.8 \times 10^{18} \mathrm{~cm}^{-3}$, respectively. The jet length is $3 \mathrm{~mm}$. The laser power on target is $42 \mathrm{TW}$.

With the laser power and the target length fixed, the plasma density was scanned by varying the backing pressure on the nozzle until electron beams of the highest quality were produced. The position of the laser focal plane with respect to the nozzle was kept constant over the entire series of shots. Following is a detailed analysis of one representative set carried out with a $3 \mathrm{~mm}$ nozzle and 42-TW on target. Electrons produced at densities above $\mathrm{n}_{\mathrm{e} 0}=2.5 \times 10^{19} \mathrm{~cm}^{-3}$ had large divergence, continuously decaying energy spectra (not shown), and large shot-to-shot variation of parameters. Monoenergetic features first emerged at $\mathrm{n}_{\mathrm{e} 0}=2.5 \times 10^{19} \mathrm{~cm}^{-3}$. Further decrease of density caused reduction of the energy spread and charge, with a concomitant increase of the peak energy and suppression of the low-energy tail. Electron beams of the highest quality -- central energy 350$\mathrm{MeV}$, charge 5-10-pC, and no detectable low-energy background -- were generated at $\mathrm{n}_{\mathrm{e} 0}=6 \times 10^{18} \mathrm{~cm}^{-3}$. Below this density, the peak electron energy dropped sharply with no detectable signal below $\mathrm{n}_{\mathrm{e} 0}=3.5 \times 10^{18} \mathrm{~cm}^{-3}$. The evolution of single-shot energy spectra is presented in Figure 1 ; multi-shot electron beam statistics for each set of parameters are summarized in Table 1. The dark current component of the beam is quantified by computing the contrast, defined as the ratio of the charge within the FWHM of the quasi-monoenergetic peak to the total charge on the LANEX. If significant dark current is present, the contrast would be low, and vice versa. As demonstrated by the data of column $\mathrm{C}$ of Table 1 , the optimal beams have the highest contrast, $\sim 0.75$ (same as for an ideal Gaussian distribution). Hence, all the charge is contained in the monoenergetic peak, with no detectable charge at other energies -- the beam is dark-current-free. The presence of a low-energy tail noticeably reduces the contrast; at higher densities, when the beam becomes polychromatic, the contrast is the lowest. This near-threshold operating regime is scalable: an optimal density can be found for different laser powers, and acceleration lengths, leading to a stable, dark-current-free, quasi-monoenergetic electron beam in the energy range $200-400 \mathrm{MeV}$.

Table 1: Electron beam characteristics for the parameters of Figure 1averaged over 10--30 shots; $\mathrm{E}$ is the central energy in $\mathrm{MeV}$; $\Delta \mathrm{E} / \mathrm{E}$ is the normalized energy spread (FWHM); D is the FWHM divergence in mrad; $\mathrm{P}$ is the pointing stability in mrad; $\mathrm{Q}$ is the charge in pC; $\mathrm{B}$ is the brightness (per shot) in units of $10^{10}$ electrons $\mathrm{MeV}^{-1} \mathrm{~mm}^{-2} \mathrm{mrad}^{-2}$; and $\mathrm{C}$ is the ratio of the charge within FWHM in energy to the total beam charge. The measured beam charge fluctuation is $50 \%$.

\begin{tabular}{|l|l|l|l|l|l|l|l|}
\hline & $\mathbf{E}$ & $\mathbf{\Delta E} / \mathbf{E}$ & $\mathbf{D}$ & $\mathbf{P}$ & $\mathbf{Q}$ & $\mathbf{B}$ & $\mathbf{C}$ \\
\hline (a) & 185 & 0.24 & 4.3 & \pm 3 & 240 & 19 & 0.46 \\
\hline (b) & 225 & 0.21 & 3.9 & \pm 2.8 & 85 & 7.5 & 0.55 \\
\hline (c) & 280 & 0.16 & 2.7 & \pm 1.8 & 27 & 5.5 & 0.64 \\
\hline (d) & 350 & 0.07 & 2.3 & \pm 1.1 & 7 & 8.5 & 0.75 \\
\hline (e) & 200 & 0.12 & 3.1 & \pm 1.7 & 20 & 4.4 & 0.62 \\
\hline
\end{tabular}

The results imply that a self-injection threshold corresponds to laser power ten times higher than the critical power for relativistic self-focusing. In all cases presented in Figure 1, beam loading is too weak to suppress self- 
injection. Thus, the only way to terminate injection is to limit the plasma length. Reducing the plasma density slows focusing of the pulse, delaying formation of the bubble. Below the cutoff the bubble does not form during the laser transit through the plasma, and self-injection into the first bucket does not occur. At the optimal density the bubble forms before the end of the plasma, experiences minimal evolution, and creates a quasimonoenergetic electron bunch. The plasma ends before either the laser waist oscillates or pulse selfcompresses; hence, injection into the bubble does not resume, and the continuous low-energy tail does not form.

We confirm this interpretation with three-dimensional particle-in-cell (3D PIC) simulations using preionized helium plasma with a trapezoidal profile $(0.5 \mathrm{~mm}$ linear entrance, and exit ramps and a $2 \mathrm{~mm}$ plateau $)$ and a Gaussian laser pulse with $42 \mathrm{TW}$ power, central wavelength $805 \mathrm{~nm}$, duration $30 \mathrm{fs}$, and waist $13.6 \mu \mathrm{m}$. In all simulations, the plasma extends from $\mathrm{z}=0$ to $3 \mathrm{~mm}$. Nonlinear pulse evolution was studied using the quasistatic cylindrical code WAKE with test particles. We find that a position of the focal plane with respect to the plasma is critical for self-focusing, and hence the time of initiation, and termination of self-injection. Three dimensional PIC simulations using experimentally measured parameters reproduce the electron beam characteristics (energy and charge) but overestimate the beam divergence.

\section{Conclusion and future work}

We report stable production of quasi-monoenergetic, dark-current-free, 200--400 MeV electron beams from a laser plasma accelerator operating near the threshold for electron self-injection. The optimal regime is characterized by full suppression of secondary injection into the plasma bubble. This method of controlling electron beam quality is found to be scalable to higher beam energy. Future work will involve the generation of high-quality, $>1 \mathrm{GeV}$ electron beams using the $30 \mathrm{~J}$ pulses available from the recent upgrade of the DIOCLES laser system to the PW peak power level.

\section{Acknowledgements}

This work was supported by the U. S. DOE grants DE-FG02-05ER15663, and DE-FG02-08ER55000; DARPA grant FA9550-09-1-0009; DTRA grant HDTRA1-11-C-0001 and, the U. S. DHS grant 2007-DN-007-ER000702. The DIOCLES laser is supported by the AFOSR under contracts FA 9550-08-1-0232, and FA9550-07-10521 .

\section{References}

[1] S. Mangles et al., "Monoenergetic beams of relativistic electrons from intense laser-plasma interactions," Nature 431, 535-538 (2004)

[2] C. Geddes et al., "High-quality electron beams from a laser wakefield accelerator using plasma-channel guiding," Nature 431, 538-541 (2004)

[3] J. Faure et al., “A laser-plasma accelerator producing monoenergetic electron beams,” Nature 431, 541-544 (2004)

[4] W. P. Leemans et al., “GeV electron beams from a centimeter-scale accelerator,” Nat. Phys. 2, 696-699 (2006).

[5] N. A. M. Hafz et al., "Stable generation of GeV-class electron beams from self-guided laser-plasma channels," Nat. Photon. 2, 571-577 (2008).

[6] S. Kneip et al., "Near-GeVacceleration of electrons by a nonlinear plasmawave driven by a self-guided laser pulse,” Phys. Rev. Lett. 103, 035002 (2009).

[7] D. Froula et al., "Measurements of the critical power for self-injection of electrons in a laser wakefield accelerator," Phys. Rev. Lett. 103, 215006 (2009)

[8] C. E. Clayton et al., "Self-Guided Laser Wakefield Acceleration beyond 1 GeV Using Ionization-Induced Injection," Phys. Rev. Lett. 105, 105003 (2010).

[9] A. Pukhov and J. Meyer-ter-Vehn, “Laser wake field acceleration: the highly non-linear broken-wave regime,”Appl. Phys. B 74, 355361 (2002).

[10] W. Lu et al., "Generating multi-GeV electron bunches using single stage laser wakefield acceleration in a 3D nonlinear regime," Phys. Rev. ST Accel. Beams 10, 061301(2007)

[11] S. Kalmykov et al., "Electron Self-Injection and Trapping into an Evolving Plasma Bubble,” Phys. Rev. Lett. 103, 135004 (2009).

[12] S. Kneip et al., "Bright spatially coherent synchrotron X-rays from a table-top source,” Nat. Phys. 6, 980-983 (2010).

[13] J. Osterhoff et al., "Generation of stable, low-divergence electron beams by laser-wakefield acceleration in a steady-state-flow gas cell,” Phys. Rev. Lett. 101, 085002 (2008)

[14] V. Ramanathan et al., "Submillimeter-resolution radiography of shielded structures with laser-accelerated electron beams," Phys. Rev. ST Accel. Beams 13, 104701 (2010). 